Cinémas

Revue d'études cinématographiques

Journal of Film Studies

\title{
L’image au cinéma ou le corps (d)écrit
}

\section{Lucie Roy}

Volume 7, numéro 1-2, automne 1996

La Représentation du corps au cinéma

URI : https://id.erudit.org/iderudit/1000930ar

DOI : https://doi.org/10.7202/1000930ar

Aller au sommaire du numéro

\section{Éditeur(s)}

Cinémas

ISSN

1181-6945 (imprimé)

1705-6500 (numérique)

Découvrir la revue

Citer cet article

Roy, L. (1996). L’image au cinéma ou le corps (d)écrit. Cinémas, 7(1-2), 11-35. https://doi.org/10.7202/1000930ar

\section{Résumé de l'article}

Partant de la conception bergsonienne de la perception d'images qui passe par l'image du corps dans la phénoménologie quotidienne, l'auteure propose d'aborder la problématique de la perception au cinéma et, plus particulièrement, de la perception du corps au cinéma. Cette perception du corps au cinéma, si elle passe par des images, et par des images qui font corps, elle impose une réflexion quant au système de prédication qu'induit ce qui a été appelé dans le présent article le " corps-percevant » de l'écran. Dans cette première partie du texte, donc, l'auteure réfléchit, pour le cinéma, à cette notion de corps percevant, alors que dans la seconde patrie, elle se livre à une étude de la séquence librement intitulée "La scène du petit manteau rouge " qui sert de motif dans cette région du film La Liste de Schindler. Dans ce film, et dans cette scène particulièrement, se joue une véritable opération langagière de classement et de différemment des corps (c'est la « descripture " filmique) dont les enjeux idéologiques sont lourds.
Ce document est protégé par la loi sur le droit d'auteur. L'utilisation des services d'Érudit (y compris la reproduction) est assujettie à sa politique d'utilisation que vous pouvez consulter en ligne.

https://apropos.erudit.org/fr/usagers/politique-dutilisation/ 


\section{Limage au cinéma ou le corps (d)écrit ${ }^{1}$}

\section{Lucie Roy}

\section{RÉSUMÉ}

Partant de la conception bergsonienne de la perception d'images qui passe par l'image du corps dans la phénoménologie quotidienne, l'auteure propose d'aborder la problématique de la perception au cinéma et, plus particulièrement, de la perception du corps au cinéma. Cette perception du corps au cinéma, si elle passe par des images, et par des images qui font corps, elle impose une réflexion quant au système de prédication qu'induit ce qui a été appelé dans le présent article le "corps-percevant" de l'écran. Dans cette première partie du texte, donc, l'auteure réfléchit, pour le cinéma, à cette notion de corps percevant, alors que dans la seconde partie, elle se livre à une étude de la séquence librement intitulée "La scène du petit manteau rouge " qui sert de motif dans cette région du film La Liste de Schindler. Dans ce film, et dans cette scène particulièrement, se joue une véritable opération langagière de classement et de différemment des corps (c'est la "descripture " filmique) dont les enjeux idéologiques sont lourds.

\section{ABSTRACT}

Starting with the Bergsonian concept of the perception of images founded on the image of the body in everyday phenomenology, the author seeks to examine the problematic of cinematic perception, and more particularly the cinematic perception of the body. This cinematic perception of the body, while based on images, and images that have body, impels us to reflect on the system of predication entailed by what in this article is 
called the "perceiving body" of the screen. In the first section of the text, the author explores, for the cinema, this notion of the perceiving body, while in the second part, she undertakes a study of the sequence she calls

"The Scene of the Little Red Coat," referring to a motif in a segment of the film Schindler's List. In this film, and in this scene especially, there occurs a veritable linguistic operation of classification and "differment" of bodies (this is filmic "descripture") with heavy ideological stakes.

Toute entrée dans le monde des signes implique un assujettissement aux fictions de la représentation institutionnelle, à commencer par celle du langage, qui ne peut pas nommer sans classifier et différer [différencier].

Ivan Almeida

Il aurait été possible d'aborder cette étude de la représentation du corps au cinéma en jouant exclusivement, de façons diachronique et interdisciplinaire, de comparaisons entre les diverses façons de le "figurer", c'est-à-dire de le "réduire" ou, au contraire, de l'"élever" à l'état de figure. De le "réduire" dans la mesure où le corps est, par rapport à l'écriture, même filmique qui passe par des images, le perpétuel absent. De l' "élever» dans la mesure où le corps est, par rapport à l'écriture, surtout filmique qui passe par des images, l'infatigable et relatif "présent". Le terme "présent" est employé dans le sens peu courant de "donation" faite à l'image ou, si l'on préfere, de "mise en présence" du corps dans l'image du film. Le corps à l'image n'est pas "présent" mais, la différence est marquante, "fait présence».

Car, disait Claude Reichler, toute fiction interprétative aliène le corps comme tel; celui-ci reste sans doute disponible, mais aussi toujours rebelle, irréductible à sa représentation, développant une négativité, un espace propre $[\ldots]$. Cette réserve pourtant n'est guère perçue, et le sentiment dominant semble bien être celui d'une déception : la conscience, la douleur et la jouissance de notre corps nous sont dérobées par le fait qu'elles deviennent signes, images, mots, concepts (p. 2). 
Le corps textuel est toujours déjà fiction. En tant qu'il est toujours déjà fiction, il croise la remémoration (le "rappel " du corps) et l'imaginaire (l'«appel» du corps). "[...] le récit qui raconte le corps réitère une perte originaire, mais il constitue aussi l'unique voie d'une remémoration [imaginante de l'imagos]" (Reichler, p. 3).

De la peinture rupestre où le corps ou les corps étaient représentés en aplat et figuraient des postures comme les actions qu'elles induisent : combattre, chasser, pêcher, porter..., à la peinture de la Renaissance italienne, où les corps étaient livrés à d'autres postures, celles-là propices à l'étude des proportions et de la perspective plus qu'à l'étude, pourrait-on dire et comme c'était le cas pour la peinture rupestre, d'actions à portée "symbolique" telles que combattre, chasser, pêcher, porter..., jusqu'à ce que Bruchon-Schweitzer et Maisonneuve appelaient la "désidéalisation" du corps qui a prévalu au siècle dernier, les représentations du corps ont subi maintes transfigurations esthétiques, en même temps que maintes transfigurations idéologiques. L'on est effectivement passé, comme en ont témoigné Bruchon-Schweitzer et Maisonneuve, du "corps choyé" de Renoir au "corps disgracié" de Manet, au "corps traqué " de Degas (p. 152), au "corps disloqué" de Picasso, au "corps méconnaissable ou violé" de Duchamp, au "corps démembré" de Adzak, au "corps laboratoire de la violence" de Schwarzkogler, au " corps à retrouver " de Delay ${ }^{2}$.

Au cinéma, et dans le cinéma des premiers temps particulièrement, comptait grosso modo l'enregistrement de ce qui a été appelé la phénoménologie quotidienne. Les personnages, corps dansants de l'écran, étaient en contact avec des objets, justement, de la vie quotidienne. Dans Arrivée d'un train en gare de La Ciotat et Bataille de boules de neige des frères Lumière pour ne donner que deux exemples —, comptaient, en effet, cette arrivée du train et cette bataille avec des boules de neige. Comme dans les peintures rupestres, le cinéma des premiers temps procédait à la figuration d'actions, non plus par l'emploi de postures qui auraient pu les évoquer, mais par l'enregistrement de ces actions mêmes: attendre, s'avancer vers le train ou en sortir, se battre avec des boules de neige - ou, en référence à 
d'autres films Lumière, démolir un mur (Démolition d'un mur), pêcher des crevettes (Enfants pêchant des crevettes).

Plus récemment, dans le cinéma où sont utilisées les nouvelles technologies, on assiste à une sorte de dilution du corps, de disparition des rapports du corps avec des objets ou, si l'on veut, à l'apparition du corps-objet ${ }^{3}$, pour lequel les formes sont si manipulables et si manipulées que l'identité du corps s'en trouve profondément altérée. L'action, ici, vise la transformation même de l'image, celle du corps par des images changeantes et non, par exemple, la mise en image de la phénoménologie quotidienne.

Bref, les nouvelles technologies paraissent être à l'écriture et à son matériau ce que les peintures rupestres étaient aux postures dont on a dit qu'elles induisaient des actions: combattre, chasser, pêcher, porter. Ou plutôt les actions, en ce qui concerne les nouvelles technologiques, s'attachent à la figuration dans un même plan des transformations successives que subit l'image. Ces actions, ces transformations participent du "pro-jet ${ }^{4}$ ", c'està-dire de la projection de l'écriture même, en ses capacités et ses limites. Les nouvelles technologies ne viseraient non pas l'enregistrement des actions ou de l'"action humaine" - pour reprendre ce qui, curieusement, paraît être une ancienne expression. L'«action humaine" y concernerait, et ce n'est pas peu dire, le "penser" de l'écriture, sa "monstration" " (c'est "montrer " en général) et non, au sens philosophique du terme, la démonstration (c'est "montrer" par évidence de la preuve) ou l'indication (c'est "montrer» du doigt en quelque sorte $\left.{ }^{6}\right)$. Cette assertion indique, en termes plus simples, que le récit comptant moins, l'écriture, le matériau de l'écriture, compte plus. Un récit peut bien, en effet, compter pour beaucoup, il ne comptera qu'en rapport avec la monstration particulière qui prévaut dans le cinéma où sont utilisées les nouvelles technologies.

Dans la peinture rupestre, les corps n'étaient pas que dessinés en aplats sur les parois des grottes, ils l'étaient aussi de façon désordonnée. C'est donc, comme en témoigne Guy Gauthier ${ }^{7}$, que d'autres images de corps, bêtes ou hommes, pouvaient se trouver superposées les unes aux autres. Bref, il n'y avait pas là de cadre où domestiquer ces images du corps. Représentées en 
prise directe, figurées presque d'un jet, c'est-à-dire sans autre support de figuration que les parois en relief des grottes, ces images de corps étaient dénuées de "cadre». Avec la Renaissance italienne, la domestication de l'image du corps, et de sa représentation, atteint son paroxysme: le cadre est lourd, le personnage est en son centre et il est, proportionnellement, figuré par rapport à lui ${ }^{8}$.

Je disais que la transfiguration de l'une, la peinture rupestre, par rapport à l'autre, la peinture de la Renaissance, était chargée d'un poids esthétique et idéologique fort. J'ai, de plus, évoqué une seconde transformation, cinématographique celle-là, qui a eu cours dans le cinéma des premiers temps jusque dans le cinéma où les nouvelles technologies sont au service de l'image, du récit. Si l'un et l'autre des médias, peinture et cinéma, ont préservé quelques liens de parenté même ténus, ils ont en commun d'avoir subi des changements esthétiques et idéologiques.

\section{Phénoménologie de la perception au cinéma}

Me préoccupera, dans la prochaine partie du présent texte, l'examen des choix esthétiques ou des motifs phénoménologiques dont semble dépendre la perception au cinéma. Je prendrai, pour ce faire, l'" exemple" bergsonien de l'image du corps en images.

Donnez-moi $[\ldots]$ les images en général; mon corps finira nécessairement par se dessiner au milieu d'elles comme une chose distincte, puisqu'elles changent sans cesse et qu'il demeure invariable. La distinction de l'intérieur et de l'extérieur se ramènera ainsi à celle de la partie et du tout. Il y a d'abord l'ensemble des images [des choses qui sont ou dont j'ai des images]; il y a, dans cet ensemble, des "centres d'action" contre lesquels les images intéressantes semblent se réfléchir; c'est ainsi que les perceptions naissent et que les actions se préparent. Mon corps est ce qui se dessine au centre de ces perceptions; ma personne est l'être auquel il faut rapporter ces actions (Bergson, p. 46).

Si j'ai pris soin de citer longuement ce passage de l'ouvrage de Bergson, c'est que je le considère particulièrement parlant si on l'applique au cinéma. Bien qu'il faille d'une certaine façon 
recourir à la métaphore, je juxtaposerai, à des phrases de Bergson, des énoncés qui, dans mon esprit, concernent la phénoménologie de la perception au cinéma.

Donnez-moi les images en général [des images d'œuvres d'art et des images filmiques en particulier] ; mon corps finira nécessairement par se dessiner [par se représenter même par corps interposé] au milieu d'elles comme une chose distincte, puisqu'elles changent sans cesse et qu'il [ce corps interposé] demeure invariable [comme point de visibilité, comme regard-regardant, comme un corps percevant si l'on veut].

L'expression "corps percevant ", en usage en phénoménologie, évoque, pour le cinéma, au moins deux acceptions terminologiques qu'il me faut expliquer. La première s'applique à cette capacité qu'ont les objets, les images d'objets contenues dans l'espace de l'écran, de retourner à sa place, ce que Gardies appelait l'« œil antérieur ", un œil antérieur qui fait corps avec l'image du film ou, si l'on préfere, tient l'image au corps ${ }^{9}$. Pour la sémio-pragmatique, nulle perception écranique sans que ne se rencontre dans l'espace visible ou l'espace perçu, cet œil antérieur et sans que, si je veux profiler encore un peu la pensée de Gardies, cet œil antérieur ne "tombe", en quelque sorte, dans l'" œil spectatoriel ", sans que cet œil antérieur ne lui offre un parcours de lecture de l'espace ${ }^{10}$.

La seconde acception terminologique qui, comme on le constatera plus loin, n'est pas sans rapport avec le titre de l'ouvrage de Merleau-Ponty Le Visible et l'Invisible, se plie à cette pensée qui veut qu'il y ait toujours " [...] incorporation du voyant au visible $[\ldots]$ " (1964, p. 173), "du percevant au perçu" ".

Ce qu'il y a donc, ce ne sont pas des choses identiques à elles-mêmes qui, par après, s'offriraient au voyant, et ce n'est pas un voyant, vide d'abord, qui, par après, s'ouvrirait à elles, mais quelque chose dont nous ne saurions être plus près qu'en le palpant du regard, des choses que nous ne saurions rêver de voir "toutes nues", parce que le regard même les enveloppe, les habille de sa chair (1964, p. 173).

La déduction paraît aisée. Pour la phénoménologie, il n’y a, si je puis dire pour en ébaucher une explication, nulle perception 
sans cette rencontre du voyant et du visible, du percevant et du perçu, sans que l'espace ne fasse, en quelque sorte, "image", sans que l'espace ne se trouve habillé par le percevant, la pensée, voire l'intentionnalité narrative.

L'écrit du visible, c'est ce qui donne corps au percevant; à l'inverse, le corps percevant, invisible à l'écran, c'est ce qui donne naissance aux images. Donnant ainsi naissance aux images, il participe de sa propre visibilité. Le visible et l'invisible - c'est, je le rappelle, le titre de l'ouvrage de MerleauPonty - travaille à l'écriture, à la perception, à la perception même a-perceptive en écriture.

Pour la phénoménologie quotidienne, selon Bergson, et pour la phénoménologie de la perception au cinéma, la distinction de l'intérieur [du vu, de ce qui, à l'intérieur de l'image, est vu] et de l'extérieur [du regardant, du corps percevant qui a été vu] se ramènera ainsi à la distinction entre de la partie et du tout. Il y a d'abord l'ensemble des images [c'est-à-dire des objets qui font "images" et une image vue dans son ensemble]; il y a, dans cet ensemble, des "centres d'action" contre lesquels les images intéressantes semblent se réfléchir [et se donner à lire de la sorte, comme si ces images intéressantes ne l'étaient que par retour, par réfraction, sur le corps percevant qui les fait voir]; c'est ainsi que les perceptions naissent [ou sont nées, d'un regard qui fait corps avec les images qu'il voit et en vertu desquelles ensuite la place du corps percevant est assignée] et que les actions se préparent [ou ont, de la sorte, été préparées, c'est-à-dire, par une perception qui est en elle-même une action, c'est-à-dire, encore, par une perception-action cristallisée, mise en creux dans une matière picturale ou photonique du cinéma qui, par conséquent, fait voir, au moins hypothétiquement, l' «intérieur» — ce qui est vu - et l'"extérieur" - le corps percevant qui a vu].

Mon corps [celui du corps percevant] est ce qui se dessine au centre de ces perceptions [intérieure et extérieure]; ma personne [comme la personne du spectateur, malgré le détour de la représentation par lequel ses perceptions passent] est l'être auquel il faut rapporter ces actions [de l'ancien regardant, ou du corps percevant dont on a dit qu'il était à demeure dans, pour ainsi dire, le "gel" pictural ou photonique du cinéma et dont 
l'ensemble définit la place, en opposition à un actuel corps percevant qui, tout à fait hors image, contemple à la fois l'intérieur - les images-objets que contient l'image - et l'extérieur de l'image - l'image-corps ou le corps percevant à partir duquel les images-objets ont été vues, sont nées].

De la longue liste de ces appels et rappels qui se convoquent mutuellement, il importe de retenir l'essentiel:

i) Devant les images d'œuvres d'art et les images filmiques en particulier, mon corps finit nécessairement par se « re-re-présenter ", en passant, tout d'abord, par ce corps interposé ou l'ancien "corps percevant" compris dans l'espace de l'image - qu'il finit par se représenter au milieu des objets-images ${ }^{12}$ dans et par rapport à cet ensemble qu'elles forment -, puis, mon corps finit par se re-présenter, en se représentant lui-même comme étant hors de l'image.

ii) La capacité du corps percevant de se situer aux centres d'action fait lire cette autre capacité : la prédicative. Devant une œuvre d'art et des images du film en particulier, le corps percevant montre non seulement quoi lire mais, au moins tendancieusement, comment lire ces centres d'action en "images intéressantes".

iii) L'image filmique est toujours déjà idéologique et elle est toujours déjà idéologique à cause de ce corps percevant qui impose, premièrement, l'action, celle de la perception, et deuxièmement, compte tenu de ce qui a été dit auparavant, la lecture des centres d'action en images intéressantes, c'est-à-dire en termes de prédication. Bref, les perceptions, qui sont aussi des actions, des actions de percevoir ou, sur le plan du matériau photo-cinématographique, d'anciennes perceptions qui ont laissé des empreintes, sont à l'origine des "images intéressantes", de la prédication des centres d'action.

\section{... et autres petites phénoménologies de la perception}

Que dire de la peinture rupestre pour laquelle notamment j'avais évoqué la possibilité d'une comparaison diachronique et interdisciplinaire? Pas de cadre, ai-je dit, où il était possible de "domestiquer " l'image et, puisque cette question est au cœur de la thématique du présent numéro, pas de cadre où il était pos- 
sible de "domestiquer" l'image du corps ou, par réfraction des images-objets sur lui, le corps percevant en travail dans l'image.

Se reconnaît plutôt une tendance à l'aplatissement des personnages, bêtes et hommes, dans l'espace de l'image et à leur superposition, d'où il ressort une perpétuelle absence ou une multiplication de points de fuite. La surface, comme le support, n'est pas cadrée et œuvrée de la sorte, c'est-à-dire qu'elle ne travaille pas à la mise en cadre du regard par point de fuite point de "vue». La surface, comme le support, n'est pas cadrée, c'est une étendue en plis, en replis, en creux, en bosses... Ces images de corps, si elles sont dénuées de cadre, sont-elles également dépourvues de "centre" de perceptions-actions, de corps percevant et de corps percevant qui fait lui-même image — de point de "vue" en somme?

Les perceptions-actions font écho à l'action de percevoir. Cette action de percevoir, rappelée dans ce que j'ai dit être le "gel" de l'image, convoque la présence d'un percevant et, au demeurant, d'un percevant qui fait corps avec l'image, d'un corps percevant. L'image, dans son ensemble, évoque, compte tenu de ce qui vient d'être dit, une certaine image de corps; or, le corps, dans l'esprit de Bergson, participe des images du monde.

Voici [pour le cinéma en l'occurrence] un système d'images que j'appelle ma perception de l'univers, et qui se bouleverse de fond en comble pour des variations légères d'une certaine image privilégiée, mon corps. Cette image occupe le centre; sur elle se règlent toutes les autres; à chacun de ses mouvements tout change, comme si l'on avait tourné un kaléidoscope (p. 20).

Dans les images de corps qui prévalent dans la peinture rupestre, il n'y a pas un corps percevant dont la place serait nettement définie à partir du cadre ou à partir d'un centre de perceptionsactions - par cadrage, point de fuite, point de "vue» - , il y aurait, plutôt, absence de cadrage, de point de fuite, de point de "vue" et, si l'on veut, il y aurait mise en présence de plusieurs corps percevants eux aussi superposés. Tout se passe, en fait, comme si des cases, à peine ou pas ébauchées du tout, d'une 
bande dessinée, sans autre récit que celui de la vue, laissaient à penser que des "auteurs" différents font en sorte que les personnages changent de cases ou, plutôt, d'endroits où être "surfacés"; comme si la surface sur laquelle s'inscrivent ces images de corps suffisait à dire l'invariable. Ces dessins, qui sont plus affaire de collectivité (de séries) que d'individualité (de "pièces»), travaillent au support et à la surface plus qu'au cadre, au perçu davantage qu'au percevant, en quelque sorte. Ou plutôt le percevant a pour correspondance, non pas un "je», duquel découlerait un "objet" visuel (hommes ou bêtes) qui lui serait subordonné, mais "quelqu'un", un "qui " qui serait placé devant son vis-à-vis, hommes ou bêtes - ce qui rappelle quelque peu les travaux de Hannah Arendt ${ }^{13}$.

Au cinéma, toutefois, les centres d'action et de prédication de l'action par perceptions-actions different de ceux qui prévalaient dans la peinture rupestre et ils different à cause du statut et de la position qu'occupe le corps percevant. Ils different, en fait, dans la mesure où le cinéma $\mathrm{a}$, à peu de chose près, récupéré, de la Renaissance italienne, le cadre, celui d'un support sur lequel inscrire l'image, de même que le "centre", celui par lequel, à l'intérieur du cadre, opèrent le cadrage, le point de fuite, le point de "vue». Au cinéma, donc, la perception d'origine, le "centre" de la perception, à la fois invisible et visible, s'inscrit, au moins hypothétiquement, à l'écran: c'est le corps percevant.

Une longue explication s'impose. J'emprunte cette fois, à la réflexion d'André Gardies, les propositions qu'il a faites.

Cette solidarité, par symétrie, du regard (de l'œil spectatoriel) et du point de fuite principal se maintient quelles que soient les variations d'angle de prise de vue. Ainsi à l'objet montré en contre-plongée et situé audelà de l'écran répond, en deçà, un œil spectatoriel en position basse et symétrique. Avec la plongée, les positions s'inversent $[\ldots]$. Un principe général découle de cela: si le point de fuite (avec l'image photographique un seul point de fuite optique par plan fixe, du moins en régime représentatif ordinaire) localise l'œil spectatoriel par rapport au plan de symétrie de l'écran, toute variation du point de fuite entraîne une égale variation dans la situation de l'œil spectatoriel (p. 26-27). 
Voilà, exprimées autrement, des préoccupations plus ou moins apparentées à celles qu'avait Bergson. Elles le sont "autrement " parce que, comme on le sait, Bergson se préoccupait de l'espace et de sa perception (de la Matière et de la mémoire qui le et la traverse), tandis que Gardies s'attache à la description de la perception de l'espace au cinéma, à son écriture et, comme on vient de le voir, à sa lecture. Pour ce qui est de l'espace bergsonien je dis "espace" alors qu'il faudrait bien plutôt dire "image" pour ce qui est, donc, de l'espace bergsonien, compte le centre de la perception, le corps, qui perçoit et qui, percevant, se souvient ou le corps qui, étant image, participe aux images de monde. Pour ce qui est encore de l'espace gardiesien - je dis "espace" alors que l'espace passe par des images ou devient image -, pour ce qui est donc de l'espace gardiesien, compte un centre de la perception, celui dans lequel s'enfile, si l'on veut et dans une sorte de rapport d' " identité imaginaire", l'autre percevant qu'est le spectateur. Si l'une de ces conceptions d'espace est phénoménologique, l'autre est systémique (sémio-pragmatique en vérité), tandis que l'autre encore, la mienne, rappelle ou tend à retenir le "phénoménologique" dans le "sémiotique"; or, c'est ce rappel de l'un par l'autre qui, par classement et différemment, fait sens pour le langage, l'écriture, le récit.

La problématique qui prévaut dans la description de l'espace de la perception chez Gardies concerne la "solidarité, par symétrie, du regard (de l'œil spectatoriel) et du point de fuite». Cette symétrie, changeante au cinéma, cette symétrie produite par la position du corps percevant par rapport à l'image, si elle laisse soupçonner une évolution esthétique depuis le cinéma des premiers temps (où la caméra, immobile, imposait une symétrie fixe du regard spectatoriel et du point de fuite) jusqu’au cinéma actuel (où la caméra, mobile, impose, par rapport à ces derniers, de perpétuels changements), elle est susceptible d'indiquer une évolution idéologique qu'il importera d'étudier en dernière analyse.

\section{"La scène du petit manteau rouge ${ }^{14}$ "}

Chacun connaît le film La Liste de Schindler, chacun a forcément remarqué "La scène du petit manteau rouge " pour laquelle 


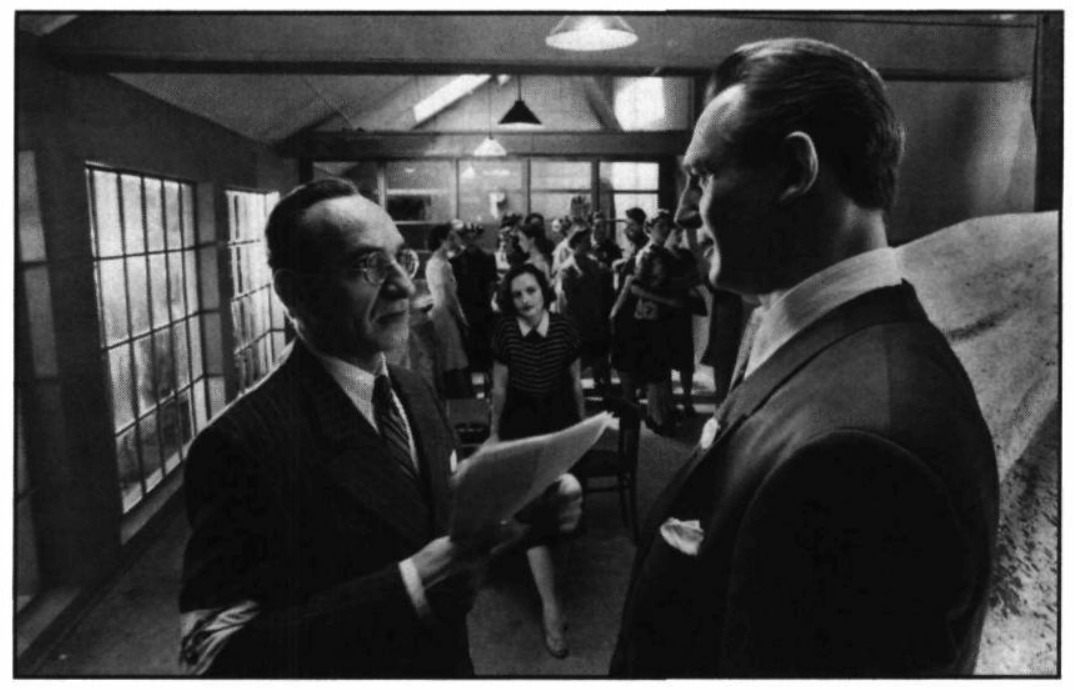

\section{Schindler's List de Steven Spielberg (1993)}

je procéderai — si une telle opération est même possible — à une sorte de réduction phénoménologique, c'est-à-dire, à cause de ce que je viens de dire, sémiotique et ce, par classement et différemment - pour conserver l'exergue du présent texte.

Avant, donc, d'entreprendre la description de la séquence dont je souhaite parler, j'aimerais dire un peu ce qu'est la réduction, du moins telle que Merleau-Ponty en fournit l'exemple en reprenant le principe énoncé par Husserl.

Ainsi ma sensation du rouge est aperçue comme manifestation d'un certain rouge senti, celui-ci [le rouge] comme manifestation d'une surface rouge, celle-ci [la surface rouge] comme manifestation d'un carton rouge, et celui-ci [le carton rouge] enfin comme manifestation ou profil d'une chose rouge, de ce livre. Ce serait donc l'appréhension d'une certaine hylè comme signifiant un phénomène de degré supérieur [...] l'opération active de signification qui définirait la conscience, et le monde ne serait rien d'autre que la "signification monde", la réduction phénoménologique serait idéaliste, au sens d'un idéalisme transcendantal qui traite le monde comme une unité de valeur indivise entre Paul et Pierre, dans laquelle leurs perspectives se recoupent, et qui fait communiquer la "conscience de 
Pierre " et la "conscience de Paul ", parce que la perception du monde "par Pierre" n'est pas le fait de Pierre, ni la perception du monde "par Paul" le fait de Paul, mais en chacun d'eux le fait de consciences prépersonnelles dont la communication ne fait pas problème, étant exigée par la définition même de la conscience, du sens ou de la vérité (Merleau-Ponty, 1945, p. V-VI).

Pour traduire quelque peu cette réduction phénoménologique d'une sensation de rouge à un manteau rouge senti dans la séquence du film portée à l'étude, l'on pourrait dire que ce rouge senti est perçu comme manifestation d'une surface rouge, que cette surface rouge est tenue pour la manifestation d'un fragment photonique rouge et que ce fragment n'est, enfin, que la manifestation ou le profil d'une chose rouge de ce film, qui tranche avec la manifestation ou le profil de toutes les autres choses qui ne sont pas rouges. Hormis l'exception consacrée à cette fillette, la fiction, toute la fiction est en noir et blanc. La fin, hors fiction, la deuxième fin pourrait-on dire, celle où le réalisateur fait défiler quelques survivants, en principe "réels", de l'Holocauste qui ont eu la chance de profiter des bienfaits de Schindler est, elle aussi, en couleurs, mais participe manifestement d'un tout autre régime.

Dans le cas de cette "scène du petit manteau rouge ", c'est, il va sans dire, le film qui fournit la possibilité de lecture, c'est-àdire l'appréhension d'une certaine hylè ou, c'est selon, d'une certaine hylé ${ }^{15}$, la perception apparaissante, naissante d'une chose rouge, et ce, par un jeu de renvois de classement et différemment de la perception des profils de choses, des images de choses qui ne sont pas rouges. Qui plus est, cette chose, différenciée, ce n'est pas une chose, mais une petite fille vêtue d'un manteau rouge. Par rapport à la lecture, donc, cette chose rouge, cette perception de la matière en "image", ne saurait totalement différer de Pierre à Paul, d'un spectateur à l'autre. Ainsi, "l'opération active de signification qui définirait la conscience et le monde ne serait rien d'autre que la "signification monde" " filmique, car la perception du monde filmique par Pierre ou par Paul n'est ni le fait de Pierre ni de Paul, mais est présence au monde filmique qui induit son propre système de sens, de 
vérité. Ce qui, évidemment, n'exclut pas l'interprétation, fruit du travail spectatoriel. C'est, au contraire, la matière, le matériau hylétique, le matériau photonique indiciel du cinéma qui fournit le support sur lequel inscrire le récit, comme son interprétation. " [...] le rapport entre le vécu hylétique et la chose n'étant pas intentionnel [mais indiciel], c'est seulement la morphé qui nous fait "reconnaître" dans la hylé la figuration de telle chose et non de telle autre" (Derrida, 1990, p. 155). La hylé du film offre, comme on l'aura compris, le matériau de l'esquisse, du dessin du récit. "L'esquisse [conséquemment] est du vécu [de la configuration de l'expérience humaine dirait Ricœur]. Or le vécu n'est possible que comme vécu et non comme spatial» (Derrida, 1990, p. 155). Seule sa refiguration est possible comme spatiale. Il n'y a pas, au cinéma, de hylé sans dessin, par intentionnalité, du système de sens et de vérité.

C'est de cette façon que j'aimerais que soit "regardé», c'est-àdire que soit pensé le regard compris dans les images de "La scène du petit manteau rouge" du film La Liste de Schindlerscène que je m'apprête à décrire —, c'est-à-dire en convoquant, en pensée, cette sensation rouge comme manifestation d'un certain rouge senti, comme manifestation d'une surface rouge, comme manifestation d'un fragment photonique rouge et comme un profil non pas d'une "chose" mais d'une petite fille au manteau rouge, comme, finalement, une certaine hylé, une sensation, une perception (c'est la "priméité " peircienne si l'on veut), puis, comme un "corps" rouge (sa "secondéité"), un corps rouge abattu dans le contexte de l'Holocauste, comme une conscience de perception qui, aussitôt, induit un système de sens, de vérité (sa tiercéité).

Schindler et sa compagne arrivent à cheval et regardent, du haut d'un promontoire, les Juifs du ghetto qui font l'objet d'une rafle de la part des soldats allemands. Des plans alternent qui montrent les Juifs terrorisés, des soldats allemands les exécutant, les poussant, les repoussant, après les avoir classés, après, donc, avoir différencié hommes, femmes et enfants.

Une caméra à l'épaule, qui ne bénéficie donc pas du dessin rassurant, parce que plus stable, d'un trépied, s'active par avances et reculs, non pas en visant des personnages à qui on aurait 
pu conférer une identité même à peine ébauchée, mais en montrant ce que ces Juifs sont devenus dans cette séquence: des corps à abattre. Vus de loin, puis de près, vus de près pour être vus de loin, puis de loin pour être vus de près, les plans montrant les Juifs se succèdent qui renversent perpétuellement, de près en loin justement, les symétries initiales. Des Juifs meurent.

Puis, le visage de Schindler apparaît alors que, toujours du haut de son promontoire, il regarde et voit une ruelle où d'autres exécutions ont lieu. Un chant d'enfants se fait entendre. Il regarde toujours tandis qu'une petite tache rouge lentement se dessine. Une fillette, on l'apprendra plus tard, s'avance au centre de la rue, imperturbable malgré le fait que, juste derrière elle, un homme tombe sous les balles. De nouveau Schindler regarde et paraît la voir alors qu'elle poursuit son avancée. Il regarde toujours. La fillette, vêtue d'un manteau rouge, marche encadrée d'autres Juifs qu'elle a rejoints alors qu'un soldat allemand l'accompagnait. Du haut du même promontoire, un panoramique - en plan subjectif, croit-on - permet de la retrouver tandis qu'elle marche seule, en sens inverse du défilé des Juifs qui, comme on s'en doute, quittent le ghetto. Schindler regarde toujours et voit, cette fois, un groupe de Juifs abattus. Au même moment, passe derrière eux la petite fille au manteau rouge. La femme qui accompagnait Schindler le prie de quitter les lieux. Schindler, imperturbablement, persiste à épier la scène. La petite fille au manteau rouge évite un attroupement de Juifs, que des soldats font monter dans un camion pour s'enfiler dans un édifice. Elle monte l'escalier. Schindler regarde, puis quitte enfin son promontoire. La petite fille se cache sous un lit. Du rouge, le manteau passe au gris. Des soldats dans la nuit défilent dans les rues. Un homme, muni d'un stéthoscope, essaie de surprendre des Juifs qui se seraient cachés.

Beaucoup plus tard dans le film, Schindler reconnaîtra cette petite fille, et davantage son manteau rouge, alors qu'on s'apprêtera à faire brûler la dépouille de cette dernière. 
La première image, par rapport à la contraction qui a été faite "images d'images", concerne le monde luimême, le langage en quelque sorte du monde, lui-même. La seconde, " [...] comme si la visibilité qui anime le monde sensible émigrait, non pas hors de tout corps, mais dans un autre corps moins lourd, plus transparent, comme si elle changeait de chair, abandonnant celle du corps pour celle du langage, et affranchie par là, mais non délivrée, de toute condition ".

Maurice Merleau-Ponty ${ }^{16}$

J'ai fait jouer des contradictions dont il me faut préciser de nouveau la valeur.

i) Par rapport à l'écriture, il n'y a pas, ai-je dit, de véritable hylé au cinéma, car le champ de perceptions est fourni par l'appareil photo-cinématographique du film et est justement le fait de Pierre ou de Paul, du réalisateur. De même, le système de valeurs induit par le corps percevant qu'inclut l'image ou inclus dans l'image est le fait de Pierre ou de Paul. Il dessine, donc, Pierre ou Paul, le trajet du film, les images variables à partir desquelles il impose son faîte, invariable.

ii) Tandis que, pour la peinture rupestre, il n'y a pas un corps percevant, mais "quelqu'un" à l'origine de la perception, tandis encore que, dans le cinéma des premiers temps, le corps percevant est pratiquement invariable à cause de la tendance à la fixité de la caméra, il n'y a que rarement, dans le cinéma actuel, de corps percevant qui serait invariable par rapport aux objets contenus dans les images. Ce qui est invariable au cinéma c'est, donc, l'existence de ce faîte de la vision à partir duquel s'organisent les mouvements et l'absence de mouvements de la perception. Bref, une perception au cinéma est invariablement action, elle peut être aussi, variablement, pourrait-on dire, en action.

Par rapport à l'immobilité de la toile ou par rapport encore à l'immobilité des images de corps, hommes ou bêtes, surfacées de 
façon éparse, sur les parois de la grotte de Lascaux par exemple, l'image mouvante, doublement mouvante du cinéma, surprend particulièrement dans cette "scène du petit manteau rouge ". On est, il est vrai, habitués à voir défiler des plans et des séquences au cinéma. Il est d'usage d'y voir des personnages en action. Il est plus surprenant de voir émerger ce que Bergson appelait, pour marquer le rapport de l'un à l'autre, l'« extérieur" de l'image en regard de l'"intérieur" des images-objets; plus surprenant, en effet, de constater que la mouvance, en profondeur (par ruptures de montage et passages de plans éloignés à des plans rapprochés ou par des "zooms" et des "travellings" avant) plutôt qu'en largeur de la caméra (par des panoramiques ou par des "travellings" de gauche à droite) permet le perpétuel exhibie du passage de l'un (l'extérieur) dans l'autre (l'intérieur). Il est, en somme, plus surprenant de constater que compte, dans "La scène du petit manteau rouge ", le perpétuel franchissement de leur frontière. Ainsi, en plus d'offrir des images filmiques, où se croisent, ici comme ailleurs, matière - hylé - et forme - morphé, en plus d'offrir un croquis de ce qu'aura pu être l'Holocauste juif, le film fournit le dessin de sa propre écriture. L'écriture elle-même fait «image ".

Il est permis de penser que cette image de l'écriture est violente à cause justement du perpétuel franchissement de la frontière entre l'intérieur (les corps à abattre) et l'extérieur (la position du corps percevant de l'image) et que cette écriture violente trace le dessin ou se prête à la description d'une violence humaine tenue pour «indescriptible».

Donc, par cet exercice de réduction, je souhaitais souligner le fait de la hylé et de la morphé de l'image filmique : i) considérer l'" apparaissant" de l'image, ce qui, de fait, à l'intérieur de l'image apparaît: la petite fille au manteau rouge; ii) reconnâtre avec plus de vivacité le faîte des images: celui du corps percevant qui, au rythme des actions contenues dans le film force l'entrée à l'intérieur de ces images qui repésentent des corps "disgraciés", "traqués... »; iii) "lire" ces centres d'action pour ce qu'ils sont: des "images", et des images "prédiquées" par avances et reculs, c'est ce qui a été appelé la double mouvance du film; prédiquées de la sorte, ces images, même violentes, sont tenues pour «intéressantes "... 


\section{Phénoménologie de la perception ou de la description?}

Je le souligne, il n'y a apparemment pas de manifestation du corps percevant d'origine dans "La scène du petit manteau rouge ». Ou plutôt, il y a manifestation d'un corps percevant fictionnel, celui du personnage qu'est Schindler qui parait voir la scène et qui sert, conséquemment, d'émissaire au premier, au corps percevant d'origine. Cette dernière remarque fait surgir l'idée d'un "ordonnancement de perceptions". Hamon parlait plus volontiers, pour la littérature, d'ordonnancement des descriptions. Comme l'on sait qu'au cinéma une perception à l'image ou, mieux, qu'une perception faite image, induit une description simultanée des objets qui y sont compris, je n'éprouve pas de difficultés à tirer parti du terme que l'auteur a privilégié, et ce, afin de mettre en évidence le fait d'une chaîne de perceptions qui sert de prétexte "vraisemblabilisant" à "La scène du petit manteau rouge " qui nous occupe.

Je m'explique: il y a, de fait, dans ce passage du film, de perpétuels retours sur et $a u$ regard de Schindler : c'est lui qui perçoit et c'est lui l'émissaire de la perception, c'est lui qui habille le perçu de sa pensée - dans ce cas compatissante; bref, à cause de cette règle d'ordonnancement des perceptions, c'est lui, laisse-t-on croire, le corps percevant, le "je" de la perception auquel les "objets" (Juifs et Allemands) sont soumis.

Si l'ordonnancement des perceptions passe par un émissaire qui perçoit les choses qui sont à l'écran, si cet ordonnancement sert de prétexte vraisemblabilisant, et s'il faut user de ce prétexte pour imposer "la signification monde filmique", c'est que le vraisemblable, l'idée du vraisemblable est, comme cela a été maintes fois souligné ailleurs, toujours déjà idéologique, culturelle. On est conséquemment autorisés à croire que, dans «La scène du petit manteau rouge " portée à l'étude, l'ordonnancement des perceptions, celui qui sert de prétexte vraisemblabilisant et dont les motifs passent par des perceptions émissaires, est invariablement adressé au spectateur.

Ainsi, à l'aspect horizontal de l'ordonnancement des perceptions qui sert la vraisemblance du récit fait écho un autre ordonnancement, celui-ci vertical, qui correspond à l'axe communicationnel du récit où se rencontrent, non pas les personnages 
destinateurs s'adressant à des personnages destinataires, mais les destinateurs et destinataires véritables que sont les auteurs et les lecteurs ou les spectateurs. Le projet théorique de la description tel qu'il a été formulé par Hamon n'aurait pu tenir dans l'étau serré d'une charade narrative et l'examen narratologique que, de la sorte, elle aurait été susceptible de convoquer. Il fallait, de fait, sortir de la "boîte" du récit et prendre, notamment, en compte la présence du spectateur. D'où, par ailleurs, la forme générale de la transmission du savoir que Hamon propose, c'est-à-dire ${ }^{17}$

\section{auteur}

1 personnage de dest ${ }^{\text {eur }}-$ savoir -1 personnage de destaire

\section{lecteur}

Les regards qui découlent du film et qui semblent appartenir au personnage destinateur (Schindler) pour un autre, destinataire (la petite fille au manteau rouge), œuvrent à la vraisemblance du récit et non, pourrait-on dire, à celle de la perception : à la vraisemblance du récit, parce ce que c'est le fait d'existence de Schindler et de l'Holocauste que le spectateur, comme on le suppose, essaie de trouver (d)écrit, c'est-à-dire écrit parce que décrit; à l'invraisemblance de la perception, parce que l'on se demande, en effet, comment on a bien pu vouloir laisser supposer que Schindler, de si loin, du haut de son promontoire, avait pu voir de si près la petite fille au manteau rouge.

Dessinant à grands traits, c'est-à-dire naïvement, les règles de la vraisemblance, je pourrais dire que le fait d'existence de Schindler dans le film est à la fois de l'ordre du factuel et du fictionnel. Ce fait d'existence de Schindler dans le film est de l'ordre du factuel dans la mesure où Schindler a véritablement existé. Il est fictionnel dans la mesure où l'intervention de Schindler pour la survie de Juifs lors de l'Holocauste est, au moins, minimalement fictionnalisée par le récit qui narre non pas les, mais des événements et veille, conséquemment, à la sériation des événements de l'histoire; fictionnel, encore, dans la mesure où la présence filmique de Schindler qui a vécu à une autre époque que celle-ci ne saurait réalistement être "rattrapée " 
par le récit; invraisemblable, enfin, dans la mesure où le fait que Schindler soit, par endroits, à l'origine de la perception des événements passés est impossible.

Ainsi, le film La Liste de Schindler met, sournoisement j'oserais dire, en scène l'indescriptible, c'est-à-dire l'intolérable de l'Holocauste. Il le fait sournoisement, parce que le récit de la vie de Schindler, l'intrigue en somme, se prête à une autre intrigue ou, autrement dit, au jeu de la violence par la description de faits historiques indescriptibles qui intriguent.

Malgré, en effet, leur caractère indescriptible, la perception et la description qui en découle sont, je le rappelle, si fortement marquées, si fortement alimentées par le franchissement des frontières entre l'extérieur et l'intérieur de la perception des images dans "La scène du manteau rouge", que des événements de l'Holocauste s'en sont trouvés fictionnellement (d)écrits. Donc, grâce ou malgré l'ordonnancement de perceptions auquel s'est livré le réalisateur, en faisant porter le regard de l'un (corps percevant) sur le regard de l'autre (Schindler) pour la perception de l'autre (la petite fille vêtue d'un manteau rouge), il reste que, dedans (ce dedans comprend, comme on l'a vu, l'intérieur des images - ce sont les Juifs massacrés - et l'extérieur - le corps percevant) et devant l'écran (c'est le lieu du spectateur), on a l'impression que "des enfants [se trouvent] des deux côtés du fil barbelé: ceux du dedans dont le trauma [même remémoré par des perceptions fictionnalisantes] est indicible, ceux du dehors dont le traumatisme est fantasmatique mais [...] présent " (Hazan, p. 303).

Compte tenu de ce qui vient d'être dit, je ne puis clore mon propos sans souligner l'importance de la phénoménologie de la perception au cinéma et, donc, sans ponctuer cette idée de classement et du différemment des corps qui, une fois qu'elle a été reconnue dans le langage filmique, a une autre résonnance, celle-ci idéologique. Cette idée même, si l'on veut raccorder avec plus d'évidence le propos esthétique au propos idéologique, de classement de différement des corps en écriture, fait surgir cette autre idée de classement et de différemment des corps véritables. Je fais, comme on s'en doute, précisément référence à ceux des Juifs victimes d'une exécution immédiate, promis à la chambre à 
gaz ou aux équipes de travail. "C'est, disait Marguerite Duras que je cite de mémoire, parce que les Juifs n'avaient pas de terre qu'on leur a pris leur corps. "

Car, enfin, on peut bien user de prétextes et de motifs vraisemblabilisants ou, au contraire, user de prétextes invraisemblabilisants, dont l'ordonnancement des perceptions en usage dans "La scène du petit manteau rouge" qui passe par le regard de Schindler n'est qu'un exemple, et on peut bien le faire pour redire, sournoisement peut-être, l'indescriptible et l'invraisemblable (invraisemblable parce qu'intolérable) de l'Holocauste, il reste que, à toutes les fois, par la perception et la description que celle-ci induit, le film rend compte d'un rapport au monde et, précisément, d'une "perception" du monde, de l'histoire, des corps et, j'ajoute, de l'écriture et des spectateurs.

\section{L'intersubjectivisation}

La langue est par là une objectivation et une intersubjectivation des mécanismes de la cognition: elle constitue une sorte de répertoire des formes dans lesquelles nous "cognitons" non pas la langue elle-même, comme disent tautologiquement les cognitivistes, mais notre rapport au monde, à nous-mêmes et aux autres, qui peut bien sûr s'exprimer autrement que par les voies de la langue, mais toujours plus ou moins explicitement en rapport avec elles ${ }^{18}$.

Pierre Ouellet

Le présent article voulait exposer la problématique ou le questionnement de l'écriture, du corps de l'écriture, de la représentation du corps à l'écran. Les thèmes et les termes en usage concernaient, finalement, l'écriture filmique (le corps écrit et le corps percevant en particulier) et la perception ou, j'ose le dire enfin autrement, la "descripture" filmique (le corps [d] écrit), là où le corps rend compte d'une relation, d'un rapport au monde et à la pensée en présence dans l'écriture filmique. 
L'hypothèse ici posée concernait ce fait: le cinéma, participant de l'écriture, est représentatif d'une "intersubjectivation des mécanismes de la cognition ", d'un "faire-cognitif ", d'une poétique. Il porte au langage la pensée en y reportant, en y rapportant ou, en termes plus usuels, en représentant écraniquement ses liens entre la pensée et le monde. Or, ces liens se trouvent configurés dans la mise en corps et en cadre qui ordonne et hiérarchise de la sorte les lieux de leur perception.

La première démarche était, pourrait-on dire, sémio-phénoménologique: c'est l'écriture qui fait corps, et c'est le corps percevant qui y intervient. La seconde démarche était thématique ou analytique et empruntait, à l'aide de l'exemple filmique de ce que j'ai appelé "La scène du petit manteau rouge ", le sentier de la représentation, c'est-à-dire l'idée de classement et de différemment des corps, des personnages dont le poids idéologique est, historiquement d'abord et filmiquement ensuite, de taille.

Université Laval

\section{NOTES}

1 On reconnaît là une allusion à l'intitulé de la revue Corps écrit, Paris, P.U.F..

2 J'interprète ici, presque librement, des planches fournies par Marilou BruchonSchweitzer et Jean Maisonneuve dans leur ouvrage intitulé Modeles du corps et psychologie esthétique (Paris: P. U. F., 1981). La partie du chapitre intitulée "Le corps depuis cent ans (de Manet à Picasso) " est celle qui a le plus attiré mon attention.

3 Comparable aux têtes sans visage et aux corps-machines du dadaïsme (Ibid., p. 158).

4 Le terme est de Martin Heidegger que j'interprète librement.

5 André Gaudreault utilise, dans un autre sens, cette expression dans son ouvrage Du littéraire au filmique. Système du récit, Québec: Presses de l'Université Laval, 1988.

6 Voir, à ce sujet, l'ouvrage de Derrida intitulé La Voix et le phénomène (Paris, P. U. F. : 1979) et particulièrement les pages 31 et 46 , où il est plus évidemment question de ce concept.

7 Or, à ce propos Guy Gauthier, que je cite longuement à cause de la pertinence de sa réflexion, disait: "Bien qu'elle [cette forme rectangulaire de la photographie et de la peinture] nous semble aujourd'hui la seule possible, son apparition est tardive dans l'histoire des images et la plupart des civilisations, asiatique ou amérindienne notamment, ne l'ont pratiquement pas utilisée, ou alors selon des proportions totalement différentes. Nos livres d'art ont tendance à masquer cette particularité en reproduisant uniformément les œuvres, fragments de peinture rupestre ou de rouleaux chinois, dans des rectangles parfaitement étrangers à l'œuvre originale. C'est oublier que la peinture pariétale des différentes époques de la préhistoire, pour s'en tenir à un exemple qui s'étend sur quelques milliers d'années, a complètement ignoré la notion 
de cadre. Les figures de Lascaux, d'Altamira ou du Tassili empiètent les unes sur les autres avec le plus parfait mépris des limites; les chefs-d'œuvre reconnus de la peinture chinoise ou japonaise sont rarement inscrits dans un cadre, et le support luimême, $s$ 'il a parfois une forme rectangulaire, est souvent tout en hauteur ou tout en largeur, ne rappelant que de très loin notre tableau. Cette notion d'un cadre clos et strictement délimité par une trace linéaire semble donc issue de la culture occidentale, selon des processus encore mystérieux [...]. Il faut donc admettre que le cadre à forme majoritairement rectangulaire est un pur produit de la civilisation technicienne occidentale [...] Le "naturel " du rectangle est une illusion de plus à mettre au compte de la mégalomanie de notre civilisation qui décrète "naturel" ce qu'elle élabore pour son propre compte" (Vingt + Une Leçons sur l'image et le sens, Paris: Edilig, 1989, p. 14).

8 Pensant à la Renaissance italienne, ressurgissent des images des toiles de Léonard de Vinci. De fait, même les toiles qui ont été appelées "détails" aidaient à mettre en évidence le travail du cadre.

9 André Gardies faisait référence à l'expression "tout-percevant " et réfléchissait à la présence du corps du spectateur devant l'écran. Relativement à la trajectoire phénoménologique qui me préoccupe, ce corps du spectateur me semble sans rapport et l'usage de cette expression "tout-percevant " paraît bien vague. Car l'image écranique n'induit pas un tout perçu, mais la présence d'un corps qui constitue le site ou le lieu à partir duquel les images sont vues. Elle induit aussi, cette image écranique, un corps qui, percevant, fait voir tout ce qui se trouve à l'intérieur du système - y compris, dans une autre perspective, les marques invisibles de l'écriture.

10 Cette question était au cœur de mes préoccupations lors de l'écriture de ma thèse de doctorat. "L'étude du silence au cinéma devrait soulever non seulement une étude du sens et, finalement, des structures qui font sens, mais elle devrait, pensonsnous, soulever aussi une étude de son langage en tant que phénomène de la pensée où l'univers filmique serait perçu comme une conscience de l'imaginaire, une conscience mémorisante du perçu et du percevant " (Lucie Roy, Le Silence au cinéma ou les structures de l'inexistant II (thèse de doctorat), Québec: Université Laval, 1989, p. 209).

11 Francesco Casetti disait : "Le film, en somme, se donne à voir : il fonde sa propre destination - et son propre destin [ou parcours] - à la fois comme un but à atteindre et comme une surface sur laquelle rebondir " (D'un regard l'autre. Le film et son spectateur, Lyon: Presses universitaires de Lyon, 1990, p. 24).

12 Des "objets-images", c'est-à-dire des objets contenus dans l'image du film et qui sont des images.

13 Lors d'une conférence intitulée "Écriture politique et politique post-modernes: de Maurice Blanchot à Hannah Arendt", Françoise Collin parlait des implications politiques qu'entraîne cette question du "qui " ou du "je " pour lequel on sait qu'un "objet" - même du discours - est subordonné. Cette conférence a eu lieu à l'Université Laval de Québec le 25 septembre 1996.

14 Alors que j'avais déjà proposé d'écrire sur le sujet La Liste de Schindler et, plus particulièrement, sur "La scène du petit manteau rouge", j'ai appris, et ai été ravie d'apprendre, que Gilles Thérien avait utilisé une expression voisine pour étudier ce passage du film et pour se prêter, en l'occurrence à son propos, à une étude de la critique. Il faut donc se référer à son article où il disait de la scène de la "petite fille au manteau rouge" qu'elle agissait comme "[...] artefact de mise en scène qui [donne] l'impression d'une certaine conscience morale chez Schindler". " La critique et la disparition de son objet ", La Critique cinématographique, sous la direction de Michel Larouche et René Prédal, Cinémas, vol. 6, n“" 2-3, printemps 1996, p. 14 ). En ce qui me concerne, la présente étude vient à la suite d'une autre à laquelle je me suis prêtée lors d'un des colloques organisés par le centre Cerisy-la-Salle (France). Ce colloque 
qui a eu lieu en août 1995 m'a permis de comparer la fiction (La Liste de Schindler) et le documentaire (La vraie histoire d'Oskar Schindler) dans la perspective de la réeffectuation de l'Histoire par le récit. Intitulée "Le documentaire: identité du discours et altérité du "réel " ", la communication dont je parle devrait paraître sous peu.

15 "Par hylétique, Husserl entend l'analyse de la matière (hylê) — ou impression brute - d'un acte intentionnel, telle la perception, abstraction faite de la forme (morphè) qui l'anime et lui confere un sens" (l'explication est de Paul Ricœur, Temps et récit, 3. Le temps raconté, Paris: Seuil, 1985, p. 46). L'explication est provisoire et sera reprise plus loin.

16 Le Visible et l'invisible, Paris: Gallimard, 1964, p. 200.

17 Je reprends presque intégralement le paragraphe d'une note infrapaginale de l'un de mes textes intitulé "Zola et le cinéma ou l'image et le phénomène" paru dans Zola et le cinéma (Québec: Presses de l'Université Laval, 1995, p. 37-62). Il faut se référer à l'article de Hamon intitulé "Qu'est-ce qu'une description?", Poétique, n" 12 (1972), p. $465-485$.

18 Pierre Ouellet, Voir et savoir. La perception des univers du discours, Québec: Balzac, 1992, p. 74.

\section{OUVRAGES CITÉS}

Bergson, Henri. Matière et mémoire. Paris: Presses Universitaires de France, 1990.

Bruchon-Schweitzer, Marilou et Maisonneuve, Jean. Modeles du corps et psychologie esthétique. Paris: Presses Universitaires de France, 1981.

Casetti, Francesco. D'un regard l'autre. Le film et son spectateur. Lyon: Presses Universitaires de Lyon, 1990.

Collin, Françoise. «Écriture politique et politique post-modernes: de Maurice Blanchot à Hannah Arendt " (conférence), Québec: Université Laval, septembre 1996.

Derrida, Jacques. Le Problème de la genèse dans la philosophie de Husserl. Paris: P.U.F., 1990.

Derrida, Jacques. La Voix et le phénomène. Paris: P.U.F., 1979.

Gardies, André. L'Espace au cinéma. Paris: Méridiens Klincksieck, 1993.

Gaudreault, André. Du littéraire au filmique.Système du récit. Paris: Méridiens Klincksieck, 1988.

Gauthier, Guy. Vingt + une Leçons sur l'image et le sens. Paris : Edilig, 1989.

Hamon, Philippe. "Qu'est-ce qu'une description?", Poétique, n 12 (1972), p. 465485.

Hazan, Marie. "Du Non-lieu de la mémoire à la lettre: un tracé et deux livres ", Santé mentale au Québec, vol. 17, n 1 (1992), p. 300-308.

Merleau-Ponty, Maurice. Le Visible et l'invisible. Paris: Gallimard,1964.

Merleau-Ponty, Maurice. Phénoménologie de la perception. Paris: Gallimard, 1945.

Ouellet, Pierre. Voir et savoir. La perception des univers du discours. Québec: Balzac, 1992.

Reichler, Claude (direction). Le Corps et ses fictions. Paris : Minuit, 1983.

Ricœur, Paul. Temps et récit, 3. Le temps raconté. Paris: Seuil, 1985.

Roy, Lucie. "Zola et le cinéma ou l'image et le phénomène", Zola et le cinéma (sous la direction de Paul Warren). Québec: PUL, 1995a.

Roy, Lucie. "Le documentaire: identité du discours et altérité du "réel" " (conférence), Les Lumière et après: l'aventure du documentaire, France: Cerisy-la-Salle, août 1995b (sous la direction de André Gardies et Jacques Gerstenkorn). 
Roy, Lucie. Le Silence au cinéma ou les structures de l'inexistant II (thèse de doctorat). Québec: Université Laval, 1989.

Thérien, Gilles. "La critique et la disparition de son objet ", La Critique cinématographique (sous la direction de Michel Larouche et René Prédal), Cinémas, vol. 6, ne 2-3 (printemps 1996) p. 141-163. 